\title{
LICC: L-BLP25 in patients with colorectal carcinoma after curative resection of hepatic metastases-a randomized, placebo-controlled, multicenter, multinational, double-blinded phase II trial
}

Carl Christoph Schimanski ${ }^{1,21,22^{*}}$, Markus Möhler ${ }^{1}$, Michael Schön², Eric van Cutsem³, Richard Greil ${ }^{4}$, Wolf Otto Bechstein ${ }^{5}$, Susanna Hegewisch-Becker ${ }^{6}$, Götz von Wichert ${ }^{7}$, Matthias Vöhringer ${ }^{8}$, Michael Heike ${ }^{9}$, Volker Heinemann ${ }^{10}$, Marc Peeters ${ }^{11}$, Stephan Kanzler ${ }^{12}$, Stefan Kasper ${ }^{13}$, Friedrich Overkamp ${ }^{14}$, Jan Schröder ${ }^{15}$, Daniel Seehofer ${ }^{16}$, Frank Kullmann ${ }^{17}$, Bernhard Linz ${ }^{18}$, Irene Schmidtmann ${ }^{19}$, Victoria Smith-Machnow ${ }^{20}$, Ines Gockel ${ }^{21}$, Hauke Lang ${ }^{21}$ and Peter R Galle ${ }^{1}$

\begin{abstract}
Background: $15-20 \%$ of all patients initially diagnosed with colorectal cancer develop metastatic disease and surgical resection remains the only potentially curative treatment available. Current 5-year survival following ROresection of liver metastases is $28-39 \%$, but recurrence eventually occurs in up to $70 \%$. To date, adjuvant chemotherapy has not improved clinical outcomes significantly. The primary objective of the ongoing LICC trial (LBLP25 In Colorectal Cancer) is to determine whether L-BLP25, an active cancer immunotherapy, extends recurrence-free survival (RFS) time over placebo in colorectal cancer patients following R0/R1 resection of hepatic metastases. L-BLP25 targets MUC1 glycoprotein, which is highly expressed in hepatic metastases from colorectal cancer. In a phase IIB trial, L-BLP25 has shown acceptable tolerability and a trend towards longer survival in patients with stage IIIB locoregional NSCLC.
\end{abstract}

Methods/Design: This is a multinational, phase II, multicenter, randomized, double-blind, placebo-controlled trial with a sample size of 159 patients from 20 centers in 3 countries. Patients with stage IV colorectal adenocarcinoma limited to liver metastases are included. Following curative-intent complete resection of the primary tumor and of all synchronous/metachronous metastases, eligible patients are randomized 2:1 to receive either L-BLP25 or placebo. Those allocated to L-BLP25 receive a single dose of $300 \mathrm{mg} / \mathrm{m}^{2}$ cyclophosphamide (CP) 3 days before first L-BLP25 dose, then primary treatment with s.C. L-BLP25 $930 \mu \mathrm{g}$ once weekly for 8 weeks, followed by s.c. L-BLP25 $930 \mathrm{\mu g}$ maintenance doses at 6-week (years 1\&2) and 12-week (year 3) intervals unless recurrence occurs. In the control arm, CP is replaced by saline solution and L-BLP25 by placebo. Primary endpoint is the comparison of recurrence-free survival (RFS) time between groups. Secondary endpoints are overall survival (OS) time, safety, tolerability, RFS/OS in MUC-1 positive cancers. Exploratory immune response analyses are planned. The primary endpoint will be assessed in Q3 2016. Follow-up will end Q3 2017. Interim analyses are not planned.

Discussion: The design and implementation of such a vaccination study in colorectal cancer is feasible. The study will provide recurrence-free and overall survival rates of groups in an unbiased fashion.

Trial Registration: EudraCT Number 2011-000218-20

\footnotetext{
* Correspondence: schimanski@1-med.klinik.uni-mainz.de

${ }^{1}$ First Deptartment of Internal Medicine, University Medical Center (UMC),

University Hospital of Mainz, Mainz, Germany

Full list of author information is available at the end of the article
} 


\section{Background}

The primary objective of the ongoing LICC trial ( $\mathbf{L}$ BLP25 In Colorectal Cancer) is to determine whether LBLP25, an active cancer immunotherapy, extends recurrence-free survival (RFS) time over placebo in colorectal cancer pts following R0/R1 resection of hepatic metastases. Stimuvax ${ }^{\circledR}$ (BLP25 Liposome Vaccine or L-BLP25) is an investigational therapeutic cancer vaccine co-developed by Oncothyreon Canada Inc. (formerly Biomira Inc., Edmonton, Canada), and Merck KGaA, Darmstadt, Germany for the use as an active specific immunotherapy for MUC1- expressing tumors. Details of the physical, chemical and pharmaceutical properties, the nonclinical trials and effects and safety in humans have been published elsewhere 2008) [1].

Colorectal cancer is amongst the three most frequent malignancies in Western countries [2,3]. Survival is delineated by local recurrence, by lymphatic and predominantly by hematogenous dissemination [4]. Mutations in tumor suppressor genes (APC, DCC, Smad-2, Smad-4, p53) and oncogenes (K-ras) are molecular determinants occurring during the development of sporadic colorectal cancer, which was first summarized in the adenoma-carcinoma sequence described by Vogelstein et al. [5-7]. Since only $8 \%$ of colorectal cancers harbor concomitant mutations of APC, K-ras, and p53, it seems very likely that additional pathogenic alterations instrumentally mediate progression and metastasis of colorectal cancer [8].

At the time of first diagnosis, about $35 \%$ of colorectal cancer patients have distant metastases [9]. Distant metastases limited to the liver occur in $15-20 \%$ of all patients initially diagnosed with colorectal cancer [10-12]. However, only $15-20 \%$ of synchronic hepatic metastases are resected by surgery. Complete surgical resection of hepatic metastases represents the only curative option: cure is otherwise not attainable. The 5-year survival after R0-resection of liver metastases ranges between $28 \%$ to $39 \%[13,14]$. Unfortunately, recurrence rates after R0 resection of hepatic metastases peak at up to $70 \%$ in the long-term follow-up [15].

The decision as to whether metastases are to be treated by surgical resection or by other therapeutic options such as neoadjuvant chemotherapy, should be discussed and determined by interdisciplinary tumor boards [9]. The requirements for surgical resections are (i) that there is no evidence of extra-hepatic tumor, (ii) less than $70 \%$ of the liver is tumor-bearing, (iii) fewer than 3 liver veins and fewer than 7 segments of the liver are affected. Further exclusion criteria for surgical resection of liver metastases are the presence of CHILD B/C cirrhosis and/or other severe concomitant diseases [16]. Using scoring systems such as that described by Fong, the prognosis can be estimated preoperatively [17]. The existence of at least two Fong points predicts a median survival of 47 months after surgical resection of liver metastasis. However, the Fong score is only an indirect correlation of evaluated parameters ( $\mathrm{N}$-stage, size and number of metastases, preoperative CEA value and duration of disease-free interval) with the probability of incidence of organ metastasis. Organ-bound micrometastases (MM) or disseminated tumor cells (DTC) are considered precursors of metachronic solid liver metastases [18-20].

A relevant clinical problem after primary or secondary resection of hepatic metastases is the high recurrence rate, ranging up to $70 \%$ [15]. According to de Jong and colleagues, the median RFS after resection of liver metastases is 23 months andthe median OS 36 months [15]. The application of classical chemotherapeutic strategies has not been sufficiently successful. Adjuvant 5FU has non-significantly increased recurrence free survival (RFS 1.6 yrs. vs. 2.3 yrs.; $\mathrm{P}=0.06$ ), however this did not translate into a prolonged overall survival (4.0 vs. 5.0 years; $\mathrm{P}=0.09$ ) [21]. Similar data was published by Portier et al., prolonging the 5 -year-RFS $(33.5 \%$ vs. $26.7 \%$; $P=0.028$ ) without augmenting the 5 -year-survival $(51.1 \%$ vs. $41.1 \% \mathrm{P}=0.13)$ by an adjuvant 5 -FU therapy (EBM grade 2b) [22]. Parks et al. found that a 5-FU based adjuvant chemotherapy versus watch and wait significantly improved postoperative overall survival $(\mathrm{P}=$ 0.007); however these data were retrospectively analysed registered data and thus not applicable (EBM grade $3 \mathrm{~b}$ ) [23].

Nordlinger's approach using peri-operative FOLFOX non-significantly increased the 3 -yrs RFS by $7.3 \%$ (28.1\% vs. $35.4 \% ; \mathrm{P}=0.058)$; however patients treated per protocol (per protocol analysis) benefited even more $(9.2 \%$; $3-$ yrs. RFS $33.2 \%$ vs. $42.2 \%$; $P=0.025)$ (EBM grade $1 \mathrm{~b}$ ) [24]. Other studies are limited by small numbers and do not augment EBM grade 2b [25,26].

The German S3 Guideline recommends surgery of primary resectable liver metastases (recommendation grade $\mathrm{A}$; evidence grade $3 \mathrm{~b}$; strong consensus). In contrast, a neoadjuvant chemotherapy of primary resectable metastases is considered optional and is limited to welldefined, exceptional cases (recommendation grade 0; evidence grade 3 , strong consensus). Postoperative adjuvant chemotherapy after curative R0 resection of hepatic metastases is considered optional ("can be considered"; recommendation grade B; evidence grade 2, strong consensus) [9].

In contrast, according to the current consensus of the "European colorectal metastases treatment group" use of neoadjuvant/peri-operative combination chemotherapy (e.g. FOLFOX) is suggested in the case of primary 
resectable liver metastases whereas for potentially resectable liver metastases a neo-adjuvant combination chemotherapy (e.g. FOLFOX or FOLFIRI) plus application of a targeted therapy (bevacizumab, cetuximab, panitumumab) should be considered. Isolated adjuvant strategies are not recommended by the working group.

In summary, no generally accepted standard of care is available following curative-intent resection of hepatic metastases in colorectal cancer patients. L-BLP25 is a therapeutic vaccine that targets MUC1, a well known tumor-associated antigen. Recently, it has been shown that MUC1 is associated with cellular transformation as demonstrated by tumorigenicity [27] and can confer resistance to genotoxic agents [28]. High levels of MUC1 cell surface expression [29,30], reported immunosuppressive activities of its released ectodomain [31] and anti-adhesive properties [32,33] all contribute to the ability of the MUC1 antigen to protect and promote tumor cell growth and survival, making MUC1 an attractive target for cancer immunotherapy.

Based on these results, L-BLP25 has a promising potential as adjuvant therapy after curative-intent resection of hepatic metastases in colorectal cancer patients.

\section{Methods/Design}

\section{Investigational medicinal product}

L-BLP25 is a lyophilized preparation, formulated to contain $241 \mu \mathrm{g}$ of BLP25 lipopeptide and $128 \mu \mathrm{g}$ of immunoadjuvant monophosphoryl lipid $\mathrm{A}$ in a $5 \mathrm{~mL}$ glass vial. The vial also contains 13,63 mg of liposomal lipids consisting of dipalmitoyl phosphatidylcholine (DPPC), cholesterol and dimyristoyl phosphatidylglycerol (DMPG). The BLP25 lipopeptide and monophosphoryl lipid A (MPL) are associated with the lipid bilayer of the liposomes that are formed upon rehydration of the dry powder (Figure 1). The active mechanism of L-BLP25 is mediated by the entire liposome formed after reconstitution. Only the entire liposomal formulation, in which the antigen and adjuvant are integrated into the lipid bilayer, is capable of inducing the desired immune response against MUC1. L-BLP25 was designed principally to induce a cellular immune response to tumor tissues that express the MUC1 antigen. This cellular response is characterized by the proliferation of CD4positive $\mathrm{T}$ cells in response to peptide, along with production of gamma interferon and the generation of cytotoxic T lymphocytes (CTLs) capable of killing MUC1expressing tumor cells. The BLP25 lipopeptide provides the antigenic specificity for the $\mathrm{T}$ cell response, while the monophosphoryl lipid A serves as an adjuvant to enhance the cellular immune responses. The liposomal delivery system is designed to facilitate uptake of the vaccine by antigen-presenting cells (APCs) delivering the lipopeptide into the intracellular space, finally

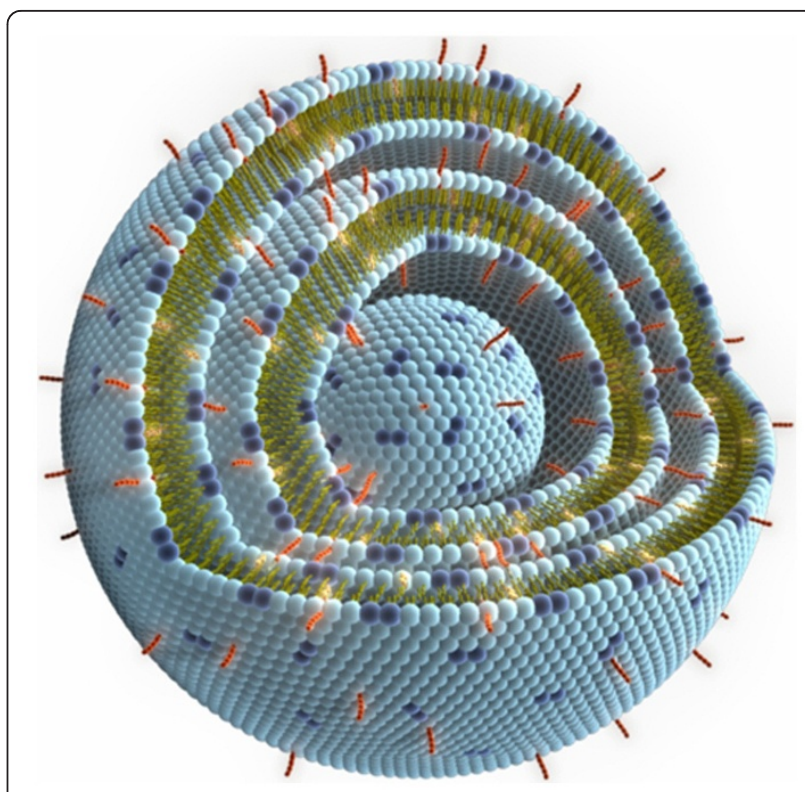

Figure 1 L-BLP25.

leading to presentation of peptides via class I and class II molecules of the HLA complex. This is expected to elicit a MUC1-specific cellular immune response mediated by $\mathrm{T}$ lymphocytes, including a CTL response. Further information on the immunological background supporting L-BLP25 and information on the immunization schedule can be found in the Investigators Brochure. Humoral responses to L-BLP25 are not expected, based on the specific formulation of the vaccine, and were found in only a few patients at low levels in previous clinical trials (EMR 63325-002, -003, and -004). Additional file 1: Table S1 provides an outline of the clinical trials conducted with L-BLP25 to date.

\section{Trial objectives}

Primary objective is the comparative evaluation of recurrence-free survival (RFS) time between the treatment groups (L-BLP25 plus cyclophosphamide versus placebo vaccination and saline infusion). Secondary objectives are the comparative assessment of overall survival (OS) time, safety/tolerability, recurrence-free survival time in the subgroup of MUC1 positive cancers, and overall survival in the subgroup of MUC1 positive cancers. MUC1 expression analysis and immuno-monitoring parameters are to be defined in separate translational protocol.

\section{Trial design and plan}

This is a randomized, placebo-controlled, multicenter, double-blinded, efficacy/safety study of L-BLP25 in patients with complete-resected metastatic CRC. Eligible patients will have had their primary tumor resected and have undergone curative-intent resection of liver 
metastases within the last 6 weeks. Eligible patients will be randomized to treatment with L-BLP25 vaccine versus placebo (2:1). Treatment will be discontinued upon documented relapse or, if subjects are free of relapse, treatment will be discontinued 3 years after the first vaccination of the maintenance phase. Randomization of 159 subjects (106 to the investigational arm and 53 to the control arm) was calculated. The enrollment period is expected to run from Q3 2011 to Q3 2013. The sample size considerations are based on the following assumptions:

- 2:1 randomization (106 pts. L-BLP25 vs. 53 pts. placebo)

- Increase in relapse-free time from 18 months under placebo to 24 months under L-BLP25 (i.e. a hazard ratio of 0.75 )

- Linearly increasing (cumulative) enrollment rate over 24 months

- 60 months of total trial duration (i.e., a minimum follow-up of 36 months after the last patient included).

- $\alpha=0.1$ (1-sided) used for planning, which translates to a 2 -sided $\alpha$-level of $20 \%$

- Power 59\%

- HR 0.75

- Lost to follow-up hazard rate 0.0027 (6\% drop out proportion at 60 months)

Follow-up will be event-driven and analysis is foreseen after approximately 113 events (recurrences) are reported unless this has still not occurred after completion of treatment of the last patient (i. e. 36 months after randomisation of the last active patient, which is approximately 60 months after initiation of the study). Based on a median RFS time of 18 months in the control arm and linearly increasing (cumulative) enrollment rate over 24 months, final analysis is projected at approximately 60 months post trial initiation. Assuming approximately $6 \%$ lost to follow-up, a total of 159 subjects (106 subjects in the L-BLP25 arm and 53 subjects in the control arm) will be enrolled to achieve the specified number of events in the scheduled follow-up time.

A Data Safety Monitoring Board (DSMB), monitoring the trial periodically, will ensure continued subject safety as well as the validity and scientific merit of the trial.

\section{Diagnosis and main inclusion and exclusion criteria}

This trial is designed for patients with metastatic colorectal carcinoma (CRC), who have undergone a complete resection of their primary tumor and recent resection of their liver metastases ( $\mathrm{R} 0$ or R1) with curative intent. Eligibility criteria for the LICC-trial are depicted in Table 1 and 2 .

\section{Pre-treatment evaluations}

After an informed consent form has been signed by the subject, following evaluations will be undertaken within 28 days prior to the first administration of L-BLP25 or placebo, and prior to the administration of cyclophosphamide or saline solution: medical history, family history of autoimmune disease, vital signs, physical examination (including height, weight and a limited neurological examination), electrocardiogram (ECG), ECOG performance status, imaging (computerized tomography/magnetic resonance imaging (CT/MRI),

Table 1 Eligibility criteria for the LICC-trial

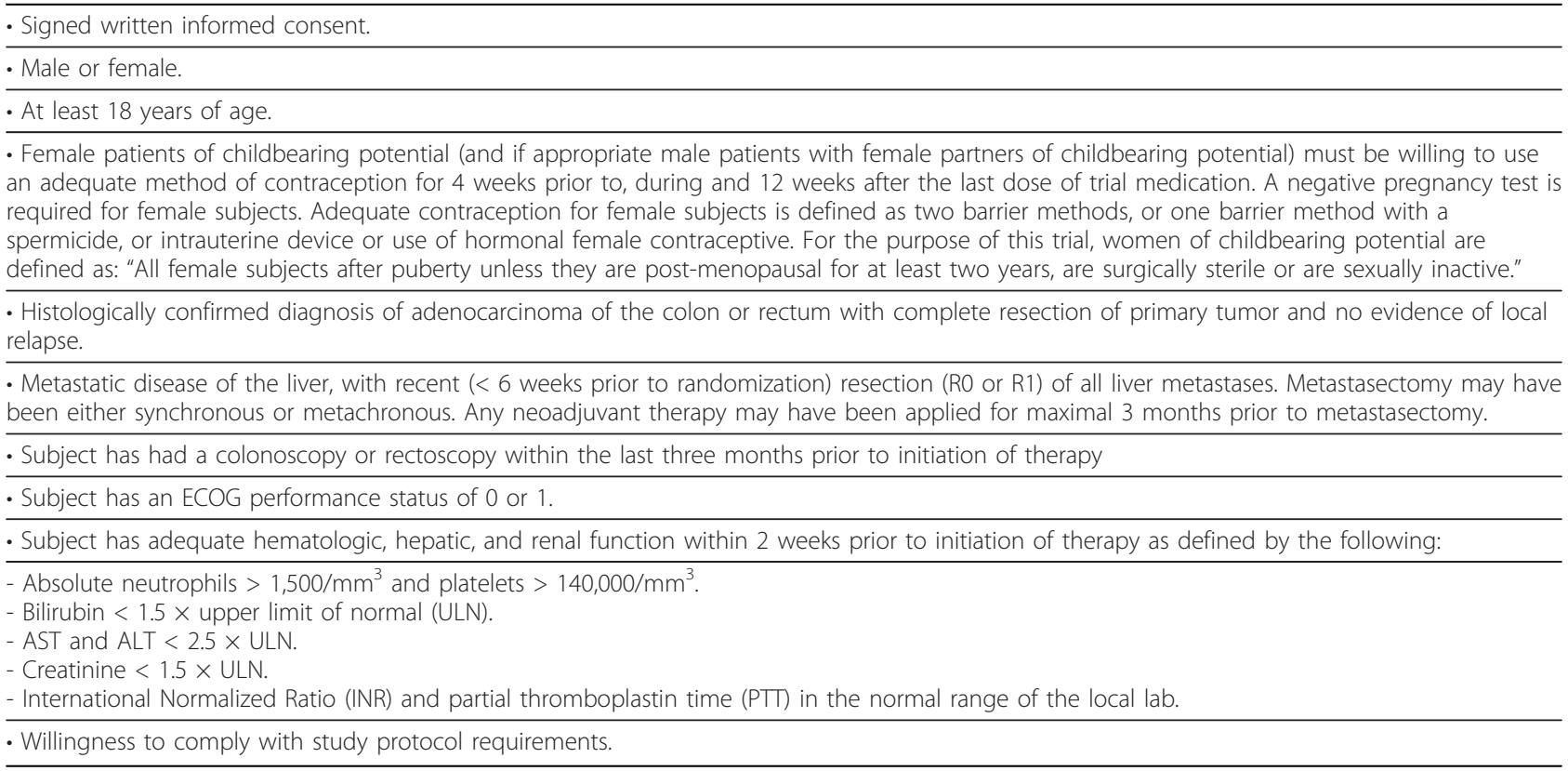

Inclusion criteria (Each of these to be met) 


\section{Table 2 Eligibility criteria for the LICC-trial}

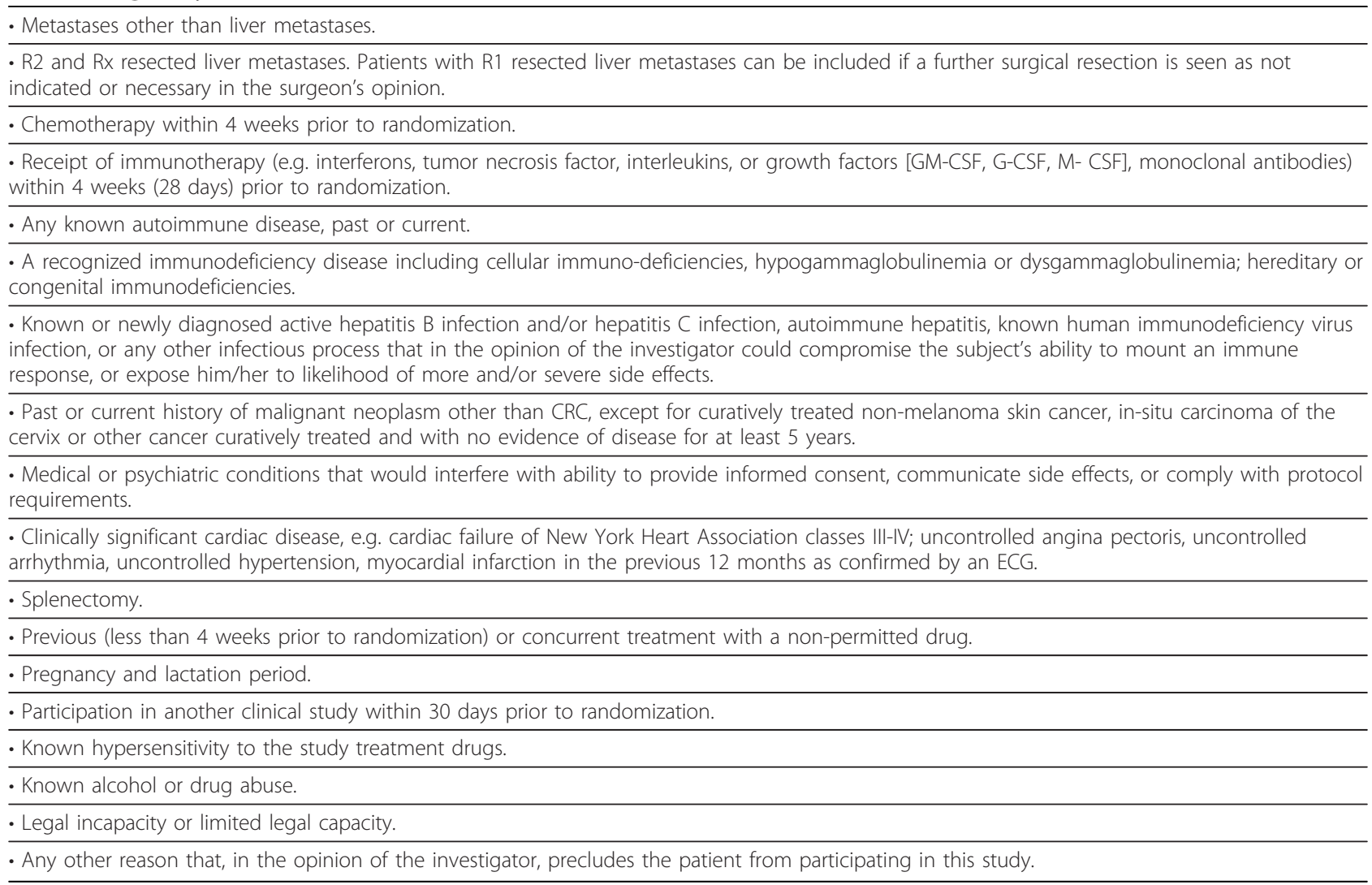

Exclusion criteria

baseline hepatic ultrasound, Fong score and pregnancy test. Within 14 days prior to the first administration of L-BLP25 or placebo and prior to administration of cyclophosphamide or placebo, blood sampling for hematology/serum biochemistry, coagulation, CEA, CA19-9, ANA, SMA, SLA, LKM, AMA, ANCA, AntiHBc-IgG, Anti-HBc-IgM, Anti-HBs, HBs antigen and anti-HCV will be performed (total blood volume for Pre-treatment evaluations: $30 \mathrm{ml}$ ). A tumor sample (paraffin fixed) must be available for immunohistochemical MUC1 analysis. The MUC1 analysis will be carried out in the central laboratory at Sponsor's site, every other standard laboratory analysis will be done in the local laboratories.

\section{Randomization}

If the subject is deemed eligible, randomization will be performed using a 2:1 ratio (Investigational arm: Placebo arm) by the help of an IVRS.

\section{Cyclophosphamide administration visit}

Within 7 days of randomization and 3 days (72 hours \pm 8 hours) prior to the first application of L-BLP25 or placebo, subjects will receive a single intravenous (I.V.) infusion of a $300 \mathrm{mg} / \mathrm{m}^{2}$ (maximum $600 \mathrm{mg}$ ) cyclophosphamide (treatment arm) or a corresponding volume of saline solution (control arm).

\section{Primary treatment phase}

Subjects will receive a target total 8 subcutaneous vaccinations of L-BLP25 or placebo at weekly intervals (primary treatment phase). Each vaccination consists of 4 subcutaneous injections. Injection sites will be inspected 30 minutes after injection.

The 5 th visit of the vaccination schedule will additionally include a physical examination, vital signs, ECOG performance status, CEA, CA19-9, ANA, hematology/ serum biochemistry and coagulation.

A hepatic ultrasound will additionally be performed at visit 8.

Evaluation after Primary Treatment Phase will take place 1 week after the completion of the primary treatment phase $\left(8^{\text {th }}\right.$ vaccination). During this visit the following tasks will be performed: physical examination, vital signs, ECG, ECOG performance status, hematology/serum biochemistry, coagulation, CEA, CA19-9 and ANA (total 30 $\mathrm{ml}$ blood volume), injection site inspection.

\section{Maintenance treatment phase}

Subjects will receive vaccinations at 6-week intervals during year 1 and 2, commencing 6 weeks after the end of the primary treatment phase. Vaccination intervals will be 12 weeks in year 3 . Subjects will receive vaccinations until documentation of recurrence or the patient 
discontinues (whatever is earlier). Treatment will end 3 years after the first vaccination of the maintenance treatment phase. Vital signs will be taken and injection sites will be inspected 30 minutes after vaccination. In addition, starting with the end of the primary treatment phase, a physical examination, ECOG performance status, CEA, CA19-9, ANA, hematology/serum biochemistry, coagulation (total $30 \mathrm{ml}$ blood volume per assessment) and a serum or urine pregnancy test (women of childbearing potential only) will be performed every 12 weeks, plus additional sampling for hematology/biochemistry only every intermediate 6 weeks until after week 92 (total blood volume $12 \mathrm{ml}$ per assessment). Vaccination intervals will be 6 weeks during years 1 and 2 after first vaccination and 12 weeks during year 3 after first vaccination.

\section{End of treatment evaluation}

When recurrence has been documented or the subject is discontinued, study treatment is to be discontinued. 12 weeks after the last treatment, an End of Treatment Evaluation will be performed, consisting of vital signs, physical examination, ECOG performance status, hematology/serum biochemistry, coagulation, ECG, CEA, CA19-9, ANA (total $30 \mathrm{ml}$ blood volume), serum or urine pregnancy test (women of childbearing potential only), CT/MRI, hepatic ultrasound and injection site inspection. When second-line treatment is indicated, and initiation is planned less than 12 weeks after the last treatment, then the end of treatment evaluation will be performed prior to the intitation of second-line therapy.

\section{Long term follow-up}

After the last treatment, i.e. after documented relapse or after the subject or investigator has decided to stop treatment, the investigator or his/her designee will contact the subject every 6 months for documentation of recurrence status and survival status. The follow-up for all patients ends 4 years after the last active patient in the maintenance treatment phase has received the first dose of the maintenance therapy (i.e. had had two such follow-up assessments). All SAEs persisting at the end of the study will be followed up until resolved/stabilized or an alternative cause is found. At the first long term follow-up 6 months after last treatment general autoimmune phenomena will be queried.

\section{Safety assessment and concomitant treatment}

$\mathrm{AE}$ assessment and recording of concomitant medication and concurrent procedures will be done during all visits in both treatment phases. During long term follow-up, AEs which are considered to be related to the investigational product will be recorded.

\section{Lab assessments and total blood volume collected}

Standard laboratory assessments include serum biochemistry, hematology, coagulation, CEA, CA19-9 and
ANA. Prior to first treatment additionally to the standard laboratory assessment ANA, SMA, SLA, LKM, AMA, ANCA, AntiHBc-IgG, Anti-HBc-IgM, anti-HBs, $\mathrm{HBs}$ antigen and anti-HCV are to determined. A planned maximum of $564 \mathrm{ml}$ blood will be drawn during the clinical trial; in the case of AE monitoring additional blood samples may be drawn. Respectively, $30 \mathrm{ml}$ per assessment will be drawn for the pre-treatment evaluation, at week 5 of the primary treatment phase, 1 week after the primary treatment phase, at 12 different time points during the maintenance treatment phase (weeks 14, 26, 38, 50, 62, 74, 86, 98, 110, 122, 134 and 146) and at the end of treatment evaluation. During the maintenance phase, additional $12 \mathrm{ml} \mathrm{sam-}$ pling per assessment for biochemistry and hematology only will be drawn at weeks $20,32,44,56,68,80$ and 92. All routine lab assessments including labeling will be performed in the local laboratories according to the local standard and local range. An additional $100 \mathrm{ml}$ blood per assessment will be drawn for the translational program in weeks $9,26,50$, and 98 in order to evaluate the immune responses (cellular/humoral) to the vaccine/placebo. A total blood volume of $500 \mathrm{ml}$ will be drawn for the translational program. Analytical test, parameters, labeling, shipment and technique of analyses for blood samples used for the translational program will be described in the separate translational program.

\section{Imaging}

CT or MRI imaging of the chest and abdomen alternating with US imaging of the liver for recurrence will be performed every 6 weeks in years 1 and 2 starting after the end of primary treatment phase, e.g. US after 8 weeks, CT after 14 weeks, US after 20 weeks. In year 3, CT or MRI imaging and alternating US imaging for relapse will be performed every 12 weeks. At study treatment termination CT/MRI and US imaging will be performed. CT/MRI will be performed according to local standard. Images will be evaluated according to Response Evaluation Criteria in Solid Tumors (RECIST 1.1) guidelines in order to guarantee uniformity. A maximum of $12 \mathrm{CT} / \mathrm{MRI}$ scans will be performed: at the pretreatment evaluations, following CT scans at weeks 14, 26, 38, 50, 62, 74, 86, 98, 122 and 146 and at the end of treatment evaluation (12 weeks after last treatment). Subjects who stop treatment prior to documentation of relapses will be required to undergo appropriate CT/MRI evaluation for detection of relapse. Subjects who withdraw from the study for clinical or symptomatic deterioration before objective documentation of relapse will be required to undergo appropriate CT/MRI to confirm relapse. Every reasonable effort will be made to ensure recurrence is confirmed. 


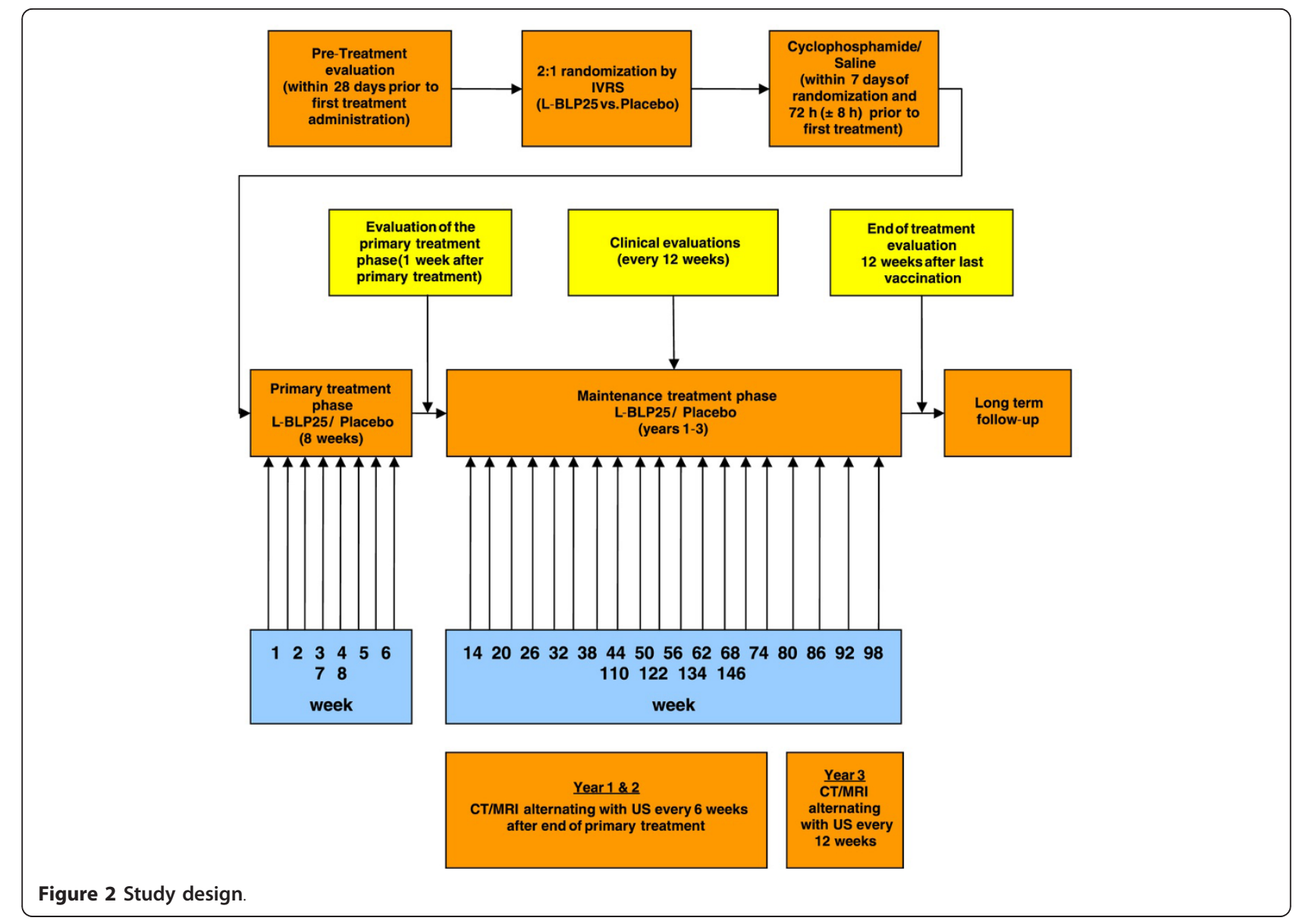

\section{Discussion}

The recurrence risk after curatively intended resection of hepatic metastases in CRC patients ranges up to 70\% [15]. No generally accepted adjuvant or perioperative strategies are available, significantly reducing the recurrence rate or prolonging overall survival [9]. According to the German S3 guideline, adjuvant chemotherapy after R0 resection of liver metastases can be considered, whereas neo-adjuvant chemotherapy prior to resection of liver metastases can be considered in reasonable exceptional cases [9]. In contrast, R0 resectable metastases limited to the liver should be resected. In summary, the recommendation for neoadjuvant/adjuvant chemotherapy is currently not strong in Germany. As the majority of centers is located in Germany we decided to comply with the German guidelines, knowing that peri-operative chemotherapy is considered standard of care in some other countries.

Participating centers confirmed the acceptance of the protocol containing a placebo arm. The standard treatment of care after resection of liver metastases in the participating centers is to watch and wait. As a consequnce, the study offers a potentially effective verum treatment to two thirds of participating patients, which would not receive any treatment otherwise.

In addition, we accept patients treated in a neoadjuvant manner, if the neoadjuvant therapy has lasted 12 weeks or less. This is necessary, as some patients have been treated in a neoadjuvant setting, but the patients are presented for the study only post surgery. As a short chemotherapy does not interfere with the postoperative immune system capacity, we agreed to include those patients. A significant impact of neoadjuvant chemotherapy has not been proven so far. Thus, the ethics committee and the Paul-Ehrlich-Institute accepted inclusion criteria.

CRC is reported to have MUC1 expression in $~ 61 \%$ of pT1, $78 \%$ of pT2, $98 \%$ of pT3 and $90 \%$ of pT4 colorectal cancer samples and thus clearly correlates with invasiveness, being an optimal target for MUC1 based vaccination strategies after resection of hepatic metastases [34]. In addition, MUC1 expression was found in 100\% (56/ 56) of colorectal cancer liver metastases [35]. A MUC1 expression of $>85 \%$ is expected in the study population analyzed, as the vast majority of metastatic cases will be $\mathrm{pT} / \mathrm{pT} 4$. It has been well discussed, that an 
engagement of the immune system might be the most successful therapeutic option to reduce micro-metastases and thus decreased recurrence rates and increase recurrence free survival time and overall survival time $[36,37]$.

L-BLP25 is designed to induce cellular cytotoxicity towards MUC1 expressing tumor cells. Doses of 1,000 $\mu \mathrm{g}$ have increased overall survival time in NSCLC patients from 13.3 months to 30.0 months in patients with stage IIIB disease without pleural effusion (HR $0.55)[38,39]$. A similar effect would be highly beneficial for patients after hepatic metastasectomy who have a high recurrence risk $(70 \%)$ with a median time to recurrence of $\sim 18-23$ months $[15,21]$.

Thus, we aim to test L-BLP-25 vaccine in a randomized, placebo-controlled, multicenter, double blinded, safety/efficacy study in patients with metastatic CRC. Eligible patients will have had their primary tumor resected and will have undergone curative-intent resection of liver metastases (R0/R1) within the last 6 weeks. Since the percentage of colorectal cancers expressing MUC1 is very high, MUC1 testing results are not required prior to inclusion. Further, the significance of detection of MUC1 expression in a tumor sample is unclear, due to the fact that this may not mean that the tumor is truly negative for MUC1, patients whose tumors are not shown to be expressing MUC1 can be included into this study. Eligible patients will be randomized via IVRS to treatment with L-BLP-25 versus placebo (2:1). Treatment will be discontinued upon documented relapse or if subjects are free from relapse, treatment will be discontinued 3 years after first application.

The dose regimen and treatment schedule will be the same as those used in Trial EMR 63325-001 (worldwide phase III, START). Throughout the development of LBLP25, a single low-dose of cyclophosphamide $(300 \mathrm{mg} /$ $\mathrm{m}^{2}$ to a maximum of $600 \mathrm{mg}$ ) has been administered prior to initiation of treatment with L-BLP25. The purpose is to overcome the immune suppression seen in cancer subjects and thus to enhance the development of an effective immune response to the MUC1 immunogen present in the vaccine.

We also decided to accept patients treated in a neoadjuvant manner, if the neoadjuvant therapy has lasted 12 weeks or less. This became necessary, as some patients have been treated in a neo-adjuvant setting, but are presented for the study evaluation only post surgery. As a short chemotherapy seems not interfere with the postoperative immune system capacity, we agreed to include those patients in order to optimize accrual.

Patients in the investigational arm receive cyclophosphamide prior to immunotherapy, in an attempt to overcome the immune suppression seen in cancer subjects. Therefore, to maintain the double-blind design, subjects in the control arm will receive saline solution in the same calculated dose volume as that of cyclophosphamide.

The design and implementation of the current vaccination study in colorectal cancer is feasible. The study will provide recurrence-free and overall survival rates of groups in an unbiased fashion.

\section{Additional material}

Additional file 1: Table S1 L-BLP25 Clinical Trials.

\section{Acknowledgements}

This study is sponsored by University Medical Center of Mainz, represented by Prof. Dr. Dr. R. Urban, Head of the Scientific Committee, University Medical Center (UMC) of Mainz, Germany. All IMPs (Investigational Medicinal Products) (L-BLP25, placebo and cyclophosphamide) are packaged and labeled for clinical trials by Merck KGaA or another suitable service provider and finally released by Merck KGaA under GMP conditions.

\section{Author details}

${ }^{1}$ First Deptartment of Internal Medicine, University Medical Center (UMC), University Hospital of Mainz, Mainz, Germany. ${ }^{2}$ Department of General and Abdominal Surgery, Municipal Hospital of Karlsruhe, Karlsruhe, Germany. ${ }^{3}$ Digestive Oncology Unit, University Hospital Gasthuisberg, Leuven, Belgium. ${ }^{4}$ Third Department of Internal Medicine, University Medical Center (UMC), University Hospital of Salzburg, Salzburg, Austria. ${ }^{5}$ Department of General and Abdominal Surgery, University Medical Center (UMC), University Hospital of Frankfurt, Frankfurt, Germany. ${ }^{6}$ Onkologische Schwerpunktpraxis Eppendorf, Hamburg, Germany. ${ }^{7}$ First Department of Internal Medicine, University Medical Center (UMC), University Hospital of Ulm, Ulm, Germany. ${ }^{8}$ Center of Internal Medicine, Robert-Bosch Hospital, Stuttgart, Germany. ${ }^{9}$ Med. Klinik Mitte, Hospital of Dortmund, Dortmund, Germany. ${ }^{10}$ Third Department of Internal Medicine, University Hospital of Großhadern, Munich, Germany. ${ }^{11}$ Department of Oncology, Antwerp University Hospital, Edegem, Belgium. ${ }^{12}$ Department of Internal Medicine, Leopoldina Hospital, Schweinfurt, Germany. ${ }^{13}$ Department of Internal Medicine, University Medical Center (UMC), University Hospital of Essen, Essen, Germany. ${ }^{14}$ Oncologianova $\mathrm{GmbH}$, Recklinghausen, Germany. ${ }^{15}$ Outpatient center for hematology and oncology, Mühlheim an der Ruhr, Germany. ${ }^{16}$ Department of General, Visceral and Transplantation Surgery, Campus Virchow Klinikum, Humboldt University, Berlin, Germany. ${ }^{17}$ First Department of Internal Medicine, Kliniken Nordoberpfalz AG, Klinikum Weiden, Germany. ${ }^{18}$ Outpatient center for hematology and oncology, Offenburg, Germany. ${ }^{19}$ Institute of Medical Biometrics, Epidemiology and Informatics (IMBEI), University Medical Center (UMC), University Hospital of Mainz, Mainz, Germany. ${ }^{20}$ iOMEDICO, Freiburg, Germany. ${ }^{21}$ Department of General and Abdominal Surgery, University Medical Center (UMC), University Hospital of Mainz, Mainz, Germany. ${ }^{22}$ First Dept. of Internal Medicine, University Medical Center (UMC), University Hospital of Mainz, Langenbeckstrasse 1, D-55131 Mainz, Germany.

\section{Authors' contributions}

CCS is the principal investigator. CCS, SM, and HL designed the study and participate in the performance and coordination of the trial. MS, EvC, RG, WOB, SHB, GVW, MV, MH, VH, SK, SK, FO, HS, DS, FK, VSM, IG, HL participate in the trial performance and patient recruitment. For trial management and monitoring VSM is appointed. IS the biostatistician responsible for the statistical planning. All authors have read and approved the final manuscript.

\section{Competing interests}

The authors declare that they have no competing interests.

Received: 1 November 2011 Accepted: 11 April 2012

Published: 11 April 2012 
References

1. Powell E, Chow LQ: BLP-25 liposomal vaccine: a promising potential therapy in non-small cell lung cancer. Expert Rev Resp Med 2008, 2(1):37-45.

2. Greenlee RT, Murray T, Bolden S, Wingo PA: Cancer statistics, 2000. CA Cancer J Clin 2000, 50:7-33.

3. Weir HK, Thun MJ, Hankey BF, et al: Annual report to the nation on the status of cancer, 1975-2000, featuring the uses of surveillance data for cancer prevention and control. J Natl Cancer Inst 2003, 95:1276-1299.

4. August DA, Ottow RT, Sugarbaker PH: Clinical perspective of human colorectal cancer metastasis. Cancer Metastasis Rev 1984, 3:303-324.

5. Fearon ER, Vogelstein B: A genetic model for colorectal tumorigenesis Cell 1990, 61:759-767.

6. Cho KR, Vogelstein B: Genetic alterations in the adenoma-carcinoma sequence. Cancer 1992, 70:1727-1731.

7. Vogelstein B, Kinzler KW: The multistep nature of cancer. Trends Genet 1993, 9:138-141

8. Smith G, Carey FA, Beattie J, et al: Mutations in APC, Kirsten-ras, and p53alternative genetic pathways to colorectal cancer. Proc Natl Acad Sci USA 2002, 99:9433-9438.

9. Schmiegel W, Pox C, Reinacher-Schick A, et al: S3 guidelines for colorectal cancer. Z Gastroenterol 2010, 48:65-136.

10. Leonard GD, Brenner B, Kemeny NE: Neoadjuvant chemotherapy before liver resection for patients with unresectable liver metastases from colorectal carcinoma. J Clin Oncol 2005, 23:2038-2048.

11. Jemal A, Siegel R, Ward E, Murray T, Xu J, Thun MJ: Cancer statistics, 2007. CA Cancer J Clin 2007, 57:43-66.

12. Pawlik TM, Choti MA: Surgical therapy for colorectal metastases to the liver. J Gastrointest Surg 2007, 11:1057-1077.

13. Kato T, Yasui $K$, Hirai $T$, et al: Therapeutic results for hepatic metastasis of colorectal cancer with special reference to effectiveness of hepatectomy: analysis of prognostic factors for 763 cases recorded at 18 institutions. Dis Colon Rectum 2003, 46:S22-S31.

14. Fong $Y$, Cohen AM, Fortner JG, et al: Liver resection for colorectal metastases. J Clin Oncol 1997, 15:938-946.

15. de Jong MC, Pulitano C, Ribero D, et al: Rates and patterns of recurrence following curative intent surgery for colorectal liver metastasis: an international multi-institutional analysis of 1669 patients. Ann Surg 2009, 250:440-448

16. Poston GJ, Adam R, Alberts $S$, et al: OncoSurge: a strategy for improving resectability with curative intent in metastatic colorectal cancer. J Clin Oncol 2005, 23:7125-7134

17. Fong Y, Fortner J, Sun RL, Brennan MF, Blumgart LH: Clinical score for predicting recurrence after hepatic resection for metastatic colorectal cancer: analysis of 1001 consecutive cases. Ann Surg 1999, 230:309-318, discussion 18-21.

18. Schimanski CC, Linnemann U, Berger MR: Sensitive detection of K-ras mutations augments diagnosis of colorectal cancer metastases in the liver. Cancer Res 1999, 59:5169-1575.

19. Linnemann U, Schimanski CC, Gebhardt C, Berger MR: Prognostic value of disseminated colorectal tumor cells in the liver: results of follow-up examinations. Int J Colorectal Dis 2004, 19:380-386.

20. Schimanski CC, Linnemann U, Arbogast R, Berger MR: Extended staging results from the detection of isolated tumor cells in the liver of colorectal cancer patients. Oncol Rep 2001, 8:185-188.

21. Mitry E, Fields AL, Bleiberg $H$, et al: Adjuvant chemotherapy after potentially curative resection of metastases from colorectal cancer: a pooled analysis of two randomized trials. J Clin Oncol 2008, 26:4906-4911.

22. Portier $G$, Elias D, Bouche $O$, et al: Multicenter randomized trial of adjuvant fluorouracil and folinic acid compared with surgery alone after resection of colorectal liver metastases: FFCD ACHBTH AURC 9002 trial. $J$ Clin Oncol 2006, 24:4976-4982.

23. Parks R, Gonen M, Kemeny N, et al: Adjuvant chemotherapy improves survival after resection of hepatic colorectal metastases: analysis of data from two continents. J Am Coll Surg 2007, 204:753-761, discussion 61-63.

24. Nordlinger B, Sorbye $H$, Glimelius B, et al: Perioperative chemotherapy with FOLFOX4 and surgery versus surgery alone for resectable liver metastases from colorectal cancer (EORTC Intergroup trial 40983): a randomised controlled trial. Lancet 2008, 371:1007-1016.

25. Wein A, Riedel C, Bruckl W, et al: Neoadjuvant treatment with weekly high-dose 5-Fluorouracil as 24-hour infusion, folinic acid and oxaliplatin in patients with primary resectable liver metastases of colorectal cancer. Oncology 2003, 64:131-138.

26. Lorenz M, Muller HH, Staib-Sebler E, et al: Relevance of neoadjuvant and adjuvant treatment for patients with resectable liver metastases of colorectal carcinoma. Langenbecks Arch Surg 1999, 384:328-338.

27. Li Y, Liu D, Chen D, Kharbanda S, Kufe D: Human DF3/MUC1 carcinomaassociated protein functions as an oncogene. Oncogene 2003 22:6107-6110

28. Ren J, Agata N, Chen D, et al: Human MUC1 carcinoma-associated protein confers resistance to genotoxic anticancer agents. Cancer Cell 2004, 5:163-175.

29. Ho SB, Niehans GA, Lyftogt $C$, et al: Heterogeneity of mucin gene expression in normal and neoplastic tissues. Cancer Res 1993, 53:641-651.

30. Zotter S, Hageman PC, Lossnitzer A: Tissue and tumor distribution of human polymorphic epithelial mucin. Cancer Reviews 1988, 11:55-101.

31. Agrawal B, Krantz MJ, Reddish MA, Longenecker BM: Cancer-associated MUC1 mucin inhibits human T-cell proliferation, which is reversible by IL-2. Nat Med 1998, 4:43-49.

32. Wesseling J, van der Valk SW, Hilkens J: A mechanism for inhibition of Ecadherin-mediated cell-cell adhesion by the membrane-associated mucin episialin/MUC1. Mol Biol Cell 1996, 7:565-577.

33. Wesseling J, van der Valk SW, Vos HL, Sonnenberg A, Hilkens J: Episialin (MUC1) overexpression inhibits integrin-mediated cell adhesion to extracellular matrix components. J Cell Biol 1995, 129:255-65.

34. Suzuki H, Shoda J, Kawamoto T, et al: Expression of MUC1 recognized by monoclonal antibody MY.1E12 is a useful biomarker for tumor aggressiveness of advanced colon carcinoma. Clin Exp Metastasis 2004, 21:321-329.

35. Zhang $\mathrm{K}$, Tang $\mathrm{W}, \mathrm{Qu} \mathrm{X}$, et al: KL-6 mucin in metastatic liver cancer tissues from primary colorectal carcinoma. Hepatogastroenterology 2009, 56:960-963.

36. Schimanski CC, Linnemann U, Galle PR, Arbogast R, Berger MR: Hepatic disseminated tumor cells in colorectal cancer UICC stage 4 patients: prognostic implications. Int J Oncol 2003, 23:791-796.

37. Schimanski CC, Mohler M, Zimmermann T, et al: Detection of hepatic micrometastases in the context of adjuvant chemotherapy and surgery for hepatic metastases. Dtsch Med Wochenschr 2010, 135:1078-1082.

38. Butts C, Murray N, Maksymiuk A, et al: Randomized phase IIB trial of BLP25 liposome vaccine in stage IIIB and IV non-small-cell lung cancer. J Clin Oncol 2005, 23:6674-6681.

39. Thatcher $\mathrm{N}$, Heighway J: Maintenance and consolidation therapy in patients with unresectable stage III/IV non-small cell lung cancer. Oncologist 2011, 15:1034-1042.

Pre-publication history

The pre-publication history for this paper can be accessed here: http://www.biomedcentral.com/1471-2407/12/144/prepub

doi:10.1186/1471-2407-12-144

Cite this article as: Schimanski et al:: LICC: L-BLP25 in patients with colorectal carcinoma after curative resection of hepatic metastases-a randomized, placebo-controlled, multicenter, multinational, doubleblinded phase II trial. BMC Cancer 2012 12:144.

\section{Submit your next manuscript to BioMed Central and take full advantage of:}

- Convenient online submission

- Thorough peer review

- No space constraints or color figure charges

- Immediate publication on acceptance

- Inclusion in PubMed, CAS, Scopus and Google Scholar

- Research which is freely available for redistribution 